\title{
Fish consumption and subsequent change in body weight in European women and men
}

Marianne U. Jakobsen ${ }^{1 *}$, Claus Dethlefsen ${ }^{2}$, Karen M. Due ${ }^{2}$, Anne M. May ${ }^{3,4}$, Dora Romaguera ${ }^{5}$, Anne-Claire Vergnaud ${ }^{5}$, Teresa Norat ${ }^{5}$, Thorkild I. A. Sørensen ${ }^{6}$, Jytte Halkjær ${ }^{7}$, Anne Tjønneland ${ }^{7}$, Marie-Christine Boutron-Ruault ${ }^{8,9}$, Francoise Clavel-Chapelon ${ }^{8,9}$, Guy Fagherazzi ${ }^{8,9}$, Birgit Teucher ${ }^{10}$, Tilman Kühn $^{10}$, Manuela M. Bergmann ${ }^{11}$, Heiner Boeing ${ }^{12}$, Androniki Naska ${ }^{13,14,15}$, Philippos Orfanos ${ }^{13,14,15}$, Antonia Trichopoulou ${ }^{13,14,15}$, Domenico Palli ${ }^{16}$, Maria Santucci De Magistris ${ }^{17}$, Sabina Sieri ${ }^{18}$, H. B. Bueno-de-Mesquita ${ }^{4,19}$, Daphne L. van der A ${ }^{4}$, Dagrun Engeset ${ }^{20}$, Anette Hjartåker ${ }^{21}$, Laudina Rodríguez $^{22}$, Antonio Agudo ${ }^{23}$, Esther Molina-Montes ${ }^{24,25}$, José M. Huerta ${ }^{25,26}$, Aurelio Barricarte ${ }^{25,27}$, Pilar Amiano ${ }^{25,28}$, Jonas Manjer ${ }^{29}$, Elisabet Wirfält ${ }^{30}$, Göran Hallmans ${ }^{31}$, Ingegerd Johansson $^{32}$, Kay-Tee Khaw ${ }^{33}$, Nicholas J. Wareham ${ }^{34}$, Timothy J. Key ${ }^{35}$, Veronique Chajès ${ }^{36}$, Nadia Slimani $^{36}$, Elio Riboli ${ }^{5}$, Petra H. M. Peeters ${ }^{3,5}$ and Kim Overvad ${ }^{1,2}$

${ }^{1}$ Department of Epidemiology, School of Public Health, Aarhus University, Bartholins Allé 2, DK-8000 Aarhus C, Denmark

${ }^{2}$ Department of Cardiology, Center for Cardiovascular Research, Aalborg Hospital, Aarbus University Hospital, Aalborg, Denmark

${ }^{3}$ Julius Center for Health Sciences and Primary Care, University Medical Center Utrecht, Utrecht, The Netherlands

${ }^{4}$ National Institute for Public Health and the Environment (RIVM), Bilthoven, The Netherlands

${ }^{5}$ Department of Epidemiology and Biostatistics, School of Public Health, Imperial College London, London, UK

${ }^{6}$ Institute of Preventive Medicine, Copenhagen University Hospital, Copenhagen, Denmark

${ }^{7}$ The Danish Cancer Society Research Center, Copenhagen, Denmark

${ }^{8}$ Inserm, Centre for Research in Epidemiology and Population Health, U1018, Institut Gustave Roussy, Villejuif, France

${ }^{9}$ Paris South University, UMRS 1018, Villejuif, France

${ }^{10}$ Division of Cancer Epidemiology, German Cancer Research Center, Heidelberg, Germany

${ }^{11}$ German Institute of Human Nutrition, Potsdam-Rebbrücke, Germany

${ }^{12}$ Department of Epidemiology, German Institute of Human Nutrition, Potsdam-Rehbruecke, Nuthetal, Germany

${ }^{13}$ WHO Collaborating Center for Food and Nutrition Policies, Athens, Greece

${ }^{14}$ Department of Hygiene, Epidemiology and Medical Statistics, School of Medicine, University of Athens, Athens, Greece

${ }^{15}$ Hellenic Health Foundation, Athens, Greece

${ }^{16}$ Molecular and Nutritional Epidemiology Unit, Cancer Research and Prevention Institute - ISPO, Florence, Italy

${ }^{17}$ Department of Clinical and Experimental Medicine, Frederico II University, Naples, Italy

${ }^{18}$ Nutritional Epidemiology Unit, Fondazione IRCCS Istituto Nazionale dei Tumori, Milan, Italy

${ }^{19}$ Department of Gastroenterology and Hepatology, University Medical Center Utrecht (UMCU), Utrecht, The Netherlands

${ }^{20}$ Department of Community Medicine, University of Tromsø, Tromsø, Norway

${ }^{21}$ Department of Nutrition, Institute of Basic Medical Sciences, University of Oslo, Oslo, Norway

${ }^{22}$ Public Health and Participation Directorate, Health and Health Care Services Council, Asturias, Spain

${ }^{23}$ Unit of Nutrition, Environment and Cancer (UNEC), Cancer Epidemiology Research Program, Catalan Institute of

Oncology (ICO), Barcelona, Spain

${ }^{24}$ Andalusian School of Public Health, Granada, Spain

${ }^{25}$ CIBER Epidemiología y Salud Pública (CIBERESP), Barcelona, Spain

${ }^{26}$ Department of Epidemiology, Murcia Regional Health Authority, Murcia, Spain

${ }^{27}$ Public Health Institute of Navarra, Pamplona, Spain

${ }^{28}$ Public Health Division of Gipuzkoa, IIS Institute Investigation BioDonostia, Basque Governement, San Sebastian, Spain

Abbreviations: EPIC, European Prospective Investigation into Cancer and Nutrition; PANACEA, Physical Activity, Nutrition, Alcohol , Cessation of smoking , Eating out of home And obesity

*Corresponding author: Dr M. U. Jakobsen, fax +45 861315 80, email muj@soci.au.dk 
${ }^{29}$ Department of Plastic Surgery, Lund University, Skåne University Hospital Malmö, Malmö, Sweden

${ }^{30}$ Department of Clinical Sciences in Malmö/Nutrition Epidemiology, Lund University, Malmö, Sweden

${ }^{31}$ Department of Public Health and Clinical Medicine, Umeå University, Nutritional Research, Umeå, Sweden

${ }^{32}$ Department of Odontology, Umeå University, Umeå, Sweden

${ }^{33}$ Clinical Gerontology Unit, Department of Public Health and Primary Care, Institute of Public Health,

University of Cambridge, Cambridge, UK

${ }^{34}$ MRC Epidemiology Unit, Institute of Metabolic Science, Addenbrooke's Hospital, Cambridge, UK

${ }^{35}$ Cancer Epidemiology Unit, University of Oxford, Oxford, UK

${ }^{36}$ International Agency for Research on Cancer (IARC-WHO), Lyon, France

(Submitted 16 September 2011 - Final revision received 20 February 2012 - Accepted 20 February 2012 - First published online 13 April 2012)

\section{Abstract}

Fish consumption is the major dietary source of EPA and DHA, which according to rodent experiments may reduce body fat mass and prevent obesity. Only a few human studies have investigated the association between fish consumption and body-weight gain. We investigated the association between fish consumption and subsequent change in body weight. Women and men $(n 344757)$ participating in the European Prospective Investigation into Cancer and Nutrition were followed for a median of $5 \cdot 0$ years. Linear and logistic regression were used to investigate the associations between fish consumption and subsequent change in body weight. Among women, the annual weight change was 5.70 (95\% CI 4.35, 7.06), $2 \cdot 23$ (95\% CI 0.16, 4.31) and $11 \cdot 12$ (95\% CI 8.17, 14.08) g/10 g higher total, lean and fatty fish consumption per $\mathrm{d}$, respectively. The OR of becoming overweight in 5 years among women who were normal weight at enrolment was 1.02 (95\% CI 1.01, 1.02), 1.01 (95\% CI 1.00, 1.02) and 1.02 (95\% CI 1.01, 1.04) g/10 g higher total, lean and fatty consumption per d, respectively. Among men, fish consumption was not statistically significantly associated with weight change. Adjustment for potential over- or underestimation of fish consumption did not systematically change the observed associations, but the $95 \%$ CI became wider. The results in subgroups from analyses stratified by age or BMI at enrolment were not systematically different. In conclusion, the present study suggests that fish consumption has no appreciable association with body-weight gain.

\section{Key words: Body weight: Diets: Fish: Follow-up studies: n-3 PUFA}

Although obesity is known to be a disorder of energy balance, an understanding of its causes and treatment remains elusive. Experimental studies have suggested that fish consumption may play a role. Fish is the major source of long-chain $n-3$ PUFA, and adding the long-chain $n-3$ PUFA EPA (20:5n-3) and DHA (22:6n-3) to a rodent diet reduces body fat mass and prevents obesity through alterations of gene expression that increase fat oxidation and energy expenditure and reduce fat deposition ${ }^{(1)}$. Moreover, it has been shown that body fat mass was reduced and the expression of genes involved in fatty acid oxidation and energy expenditure was induced when rats were fed pollock fish protein hydrolysate, abundant in the amino acids taurine and glycine, relative to soya protein or casein ${ }^{(2)}$. However, human studies have shown conflicting results. In two follow-up studies of middle-aged women and men no appreciable associations between fish consumption and subsequent change in body weight were found ${ }^{(3,4)}$. A randomised clinical trial of young overweight or obese adults following an energy-restricted diet for 8 weeks showed that the inclusion of lean fish, fatty fish or fish oil capsules resulted in statistically significantly more weight loss than did a control diet without marine food, but among men only ${ }^{(5)}$. A specific effect of long-chain $n$-3 PUFA may explain more weight loss of the diets including fatty fish or fish oil but not more weight loss of the diet including lean fish. Thus, the findings from that study suggest that some components of fish other than long-chain $n$-3 PUFA may improve weight loss such as proteins and specific amino acids.

We investigated the association between fish consumption and subsequent change in body weight. Based on previous findings, we hypothesised that both total fish consumption and the consumption of subgroups of fish (lean and fatty fish) are negatively associated with weight change. Sex, age and BMI at enrolment were considered as potential effect modifiers.

\section{Materials and methods \\ Study design and population}

In a follow-up study, we investigated the association between fish consumption and subsequent change in body weight. The study is part of the Physical Activity, Nutrition, Alcohol, Cessation of smoking, Eating out of home And obesity (PANACEA) project, which aims at studying determinants of body weight and subsequent changes in weight in Europe using data from the European Prospective Investigation into Cancer and Nutrition (EPIC). The participants ( $n$ 521448) were recruited in twenty-three centres located in ten European countries (Denmark, France, Germany, Greece, Italy, The Netherlands, Norway, Spain, Sweden and the UK) $)^{(6,7)}$. The majority of the centres invited participants from the general population typically identified according to sex and age (Denmark, Germany, Greece, Naples and Varese in Italy, 
Bilthoven in The Netherlands, Norway, Sweden as well as Cambridge and Oxford in the UK). In other centres, various sampling frameworks were used, including: blood donors (Spain as well as Ragusa and Turin in Italy); screening clinic attendees (Florence in Italy and Utrecht in The Netherlands); persons in health insurance programmes (France). The Oxford centre invited participants with an interest in health and/or vegetarian eating habits (the Oxford health-conscious population) as well as participants from the general population (the Oxford general population). In France, Naples, Utrecht and Norway, only women were recruited. In France and Norway, the cohorts were subdivided into four and two cohorts (defined as geographical country regions), respectively. At enrolment, which took place between 1992 and 2000, information on anthropometry (height and weight), habitual diet, sociodemographic (sex, age, menopausal status and highest educational level achieved) and lifestyle (tobacco consumption, physical activity and alcohol consumption) characteristics was collected. Follow-up information on

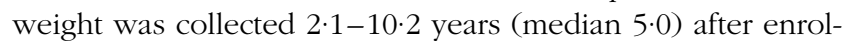
ment. No follow-up data were available from the Ragusa and Turin centres in Italy. The study was conducted according to the guidelines laid down in the Declaration of Helsinki, and all procedures involving human subjects were approved by the ethical review boards of the International Agency for Research on Cancer (Lyon in France) and all the local recruiting institutions. All participants provided written informed consent.

Among the 521448 ( $71 \%$ women) participants enrolled, persons for whom information on diet, tobacco or alcohol consumption was missing ( $n$ 6675) and persons for whom reported energy intake was extreme $(<1$ and $>99 \%$ percentile of the ratio of reported energy intake to estimated energy requirement, $n 10$ 209) were excluded, leaving 504564 participants. Between the examination at enrolment and the time of invitation to the follow-up examination, 8226 persons had died and 3991 persons had emigrated or disappeared. Among the 492347 participants eligible for invitation to follow-up, 85679 did not respond or did not want to participate. Further, 23957 persons were not yet approached for follow-up. Among the 382711 participants at follow-up, persons for whom information on follow-up time was equal to zero or missing ( $n$ 1530), persons for whom information on height or weight was missing ( $n$ 4313) and persons for whom information on anthropometric measurements was extreme (height $<130 \mathrm{~cm}$, weight $>250 \mathrm{~kg}$, BMI $<16 \mathrm{~kg} / \mathrm{m}^{2}$, waist circumference $>160 \mathrm{~cm}$, waist circumference $<60 \mathrm{~cm}$ if $\mathrm{BMI}>25 \mathrm{~kg} / \mathrm{m}^{2}$, or average weight change $>5 \mathrm{~kg} /$ year during the observation period, $n$ 2442) were excluded. Finally, pregnant women $(n$ 623) and persons with a chronic disease (prevalent diabetes, cancer or CVD) at enrolment ( $n$ 29046) were also excluded. The rationale for the exclusion of persons with a chronic disease was the likelihood of changes in dietary habits and anthropometry as a result of diagnosis and treatment. Thus, the final population consisted of 344757 persons ( $72 \%$ women). In the subgroup analyses of lean and fatty fish consumption, the Heidelberg and Potsdam cohorts in Germany, the Naples cohort in Italy and the Murcia cohort in Spain were excluded because of the lack of sufficient details in the questionnaire for classifying fish consumption into lean and fatty fish, leaving 299828 persons (74\% women) in the analyses of lean and fatty fish consumption. A flow chart of the exclusion criteria is shown in Fig. S1 (available online).

\section{Dietary assessments}

Information on habitual dietary intake was collected at enrolment through country-specific questionnaires developed and validated within the various study populations participating in the EPIC study ${ }^{(7)}$. The questionnaires were validated against twelve $24 \mathrm{~h}$ dietary recalls in seven of the ten countries ${ }^{(8)}$. Averaged over subgroups by country and sex, the correlation coefficient between the individuals' average total fish consumption estimated from the $24 \mathrm{~h}$ dietary recalls and total fish consumption estimated from the dietary questionnaires was 0.37 . Based on information from the dietary questionnaires, we investigated total fish, lean fish and fatty fish consumption. Lean fish was classified as fish with $<4 \mathrm{~g}$ fat $/ 100 \mathrm{~g}$ fish and fatty fish as fish with $4 \mathrm{~g}$ fat $/ 100 \mathrm{~g}$ fish or more ${ }^{(9)}$. In a large random sample of the participants, information on dietary intake was also collected through a standardised $24 \mathrm{~h}$ dietary recall interview using a software program (EPICSOFT) developed to standardise dietary intakes reported across the EPIC study centres ${ }^{(10,11)}$. Data collected through this method were used to account for potential measurement errors introduced by the country-specific questionnaires.

\section{Anthropometric measurements}

At enrolment, trained staff measured height and weight according to a pre-specified protocol, with participants wearing no shoes and either light underwear, light clothing or normal clothing depending on centre. Height was measured to the nearest $0.1,0.5$ or $1.0 \mathrm{~cm}$, and weight was measured to the nearest $0 \cdot 1$ or $0.5 \mathrm{~kg}$ depending on centre ${ }^{(12)}$. Weight was adjusted to reduce heterogeneity due to protocol differences in clothing worn during measurement. For participants in light clothing, the adjustment was $-1.0 \mathrm{~kg}$ and for participants in normal clothing $-1.5 \mathrm{~kg}^{(12,13)}$. At the French, Norwegian and Oxford centres, anthropometric measurements were self-measured by the participants and reported in a questionnaire. The accuracy of the self-measured weight by the participants from the Oxford health-conscious population was improved by using prediction equations derived from the Oxford general population for whom both selfmeasured weight and technician-measured weight were available $^{(14)}$. At follow-up, weight measurements were performed as at enrolment in the Cambridge centre and in the Doetinchem cohort (part of the Bilthoven centre). In the Amsterdam/Maastricht cohort (part of the Bilthoven centre) and in the other centres, the participants measured their current weight and reported in a follow-up questionnaire. The accuracy of self-measured weight by comparison with technician-measured weight has been assessed previously, and self-measured weight was found to be sufficiently accurate for identifying associations in epidemiological studies ${ }^{(14)}$. 
The outcome measure was annual change in body weight defined as weight at follow-up minus weight at enrolment divided by follow-up time. We also applied the Oxford prediction equations ${ }^{(14)}$ to all participants with self-measured weight (at enrolment and/or follow-up) and calculated corrected annual change in weight. BMI was calculated as weight in $\mathrm{kg}$ divided by height in $\mathrm{m}^{2(15)}$.

\section{Covariates}

Information on sociodemographic and lifestyle characteristics was collected through standardised questionnaires at enrolment. A four-level physical activity index (inactive, moderately inactive, moderately active and active) was derived by combining occupational physical activity with time spent on biking and other physical exercise (such as fitness training, aerobics, swimming and jogging) over the past year ${ }^{(16)}$.

\section{Statistical analyses}

The associations between fish consumption ( $10 \mathrm{~g} / \mathrm{d})$ and 1-year weight change $(\mathrm{g})$ were investigated using mixed-effects linear regression with random effects on intercept, taking into account the clustering of the data within centres nested within countries. Because of differences in the follow-up times or differences in the assessment of follow-up weight measures, the centres in Germany (Heidelberg and Potsdam), The Netherlands (Amsterdam/Maastricht, Doetinchem and Utrecht), Sweden (Malmö and Umeå) and the UK (Cambridge, Oxford general population and Oxford health-conscious population) were treated as separate centres. Analyses were carried out separately for women and men. We fit two models. The covariates in model 1 were country, centre and an indicator variable for fish consumption (consumers and non-consumers). Lean and fatty fish consumption were analysed in mutually adjusted models. The covariates in model 2 were the covariates in model 1 plus suggested risk factors for the development of obesity: age, BMI at enrolment, menopausal status (pre-, peri- and postmenopausal; women only), highest educational level achieved (no graduation, primary school, technical or professional school, secondary school, university degree and not specified), smoking status (never, former, current and unknown), physical activity level (inactive, moderately inactive, moderately active and active), total energy intake (including energy intake from alcohol consumption), and an indicator variable for the plausibility of reported energy intake (under-, acceptable- and over-reporters). Under-reporters were defined as persons with calculated values of the ratio of reported energy intake to estimated BMR below 1·14; over-reporters were defined as persons with values above $2 \cdot 10^{(17,18)}$. Adjustment for continuous variables was done using restricted cubic spline regression. For categorical variables, missing values $(1.5 \%$ women and $0.8 \%$ men for education and $13.4 \%$ women and $8.5 \%$ men for physical activity) were treated as a separate category. By stratifying the data, possible effect modification by age (classified as age at enrolment of $<60$ and $\geq 60$ years) and BMI (classified as BMI at enrolment of 18.5 to $<25,25$ to $<30$ and $\geq 30 \mathrm{~kg} / \mathrm{m}^{2}$ ) was investigated. We assessed the associations for fish consumption for non-linearity in restricted cubic spline regression models. No violations were detected (data not shown). To assess heterogeneity among the countries, country-specific (or centre, for centres with different follow-up times or differences in assessments of follow-up weight measures) estimates were calculated, and random-effects meta-analysis was used to pool these estimates.

In order to adjust for potential systematic over- or underestimation of fish consumption measurements by the dietary questionnaires, the mixed-effects linear regression analyses were rerun using predicted fish consumption data obtained from the regression of the standardised $24 \mathrm{~h}$ dietary recall interview measurements on the country-specific dietary questionnaire measurements ${ }^{(19)}$. Covariates as listed above (model 2), the season of the year in which the $24 \mathrm{~h}$ dietary recall interview was collected, and an interaction term between centre and dietary questionnaire were included in the model. The standard errors of the coefficients were estimated using bootstrap sampling (300 loops).

Secondary analyses were carried out to investigate the association between fish consumption ( $10 \mathrm{~g} / \mathrm{d})$ and the odds of becoming overweight or obese (defined as BMI $\geq 25 \mathrm{~kg} / \mathrm{m}^{2}$ ) after 5 years of follow-up using logistic regression with random effects on intercept, taking into account the clustering of the data within centres nested within countries and additionally adjusted for covariates as listed above. For these analyses, only participants who had a BMI $<25 \mathrm{~kg} / \mathrm{m}^{2}$ at enrolment were included ( $n$ 184471; 53.5\% of the study population). BMI after 5 years of follow-up was calculated using annual change in body weight and height at enrolment. A total of $15.7 \%$ of those who had a BMI $<25 \mathrm{~kg} / \mathrm{m}^{2}$ at enrolment became overweight or obese after 5 years. To assess heterogeneity among countries, country-specific (or centre) estimates were calculated, and random-effects meta-analysis was used to pool these estimates. Analyses of fish consumption and the odds of becoming overweight or obese at follow-up stratified by $<5$ or $\geq 5$ years of follow-up were also conducted.

Data analyses were performed using Stata statistical software, release 11.2 (Stata Corporation).

\section{Results}

Characteristics of the study population are shown in Table 1. Ranges of fish consumption and anthropometry by country (or centre) are shown in Table S1 (available online).

Among women, the median follow-up time was 5.0 years ( $80 \%$ central range $2 \cdot 7-9 \cdot 0$ ) and among men, the median follow-up time was $5 \cdot 2$ years ( $80 \%$ central range $2 \cdot 8-9 \cdot 6$ ).

Table 2 shows the associations between fish consumption and subsequent change in body weight. Among women, the annual weight change was $5 \cdot 70 \mathrm{~g} / 10 \mathrm{~g}$ higher total fish consumption per d (95\% CI 4.35, 7.06), 2.23 g/10 g higher lean fish consumption per d (95\% CI 0.16, 4.31) and $11 \cdot 12 \mathrm{~g} / 10 \mathrm{~g}$ higher fatty fish consumption per d (95\% CI $8 \cdot 17$, 14.08), after adjustment for potential confounders. Among men, fish consumption was not statistically significantly 
Table 1. Characteristics of the study population*

(Median values and $80 \%$ central ranges)

\begin{tabular}{|c|c|c|c|c|}
\hline & \multicolumn{2}{|c|}{ Women (n 249558) } & \multicolumn{2}{|c|}{ Men ( $n 95$ 199) } \\
\hline & Median & $80 \%$ central range & Median & $80 \%$ central range \\
\hline \multicolumn{5}{|l|}{ Physiological } \\
\hline Age (years) & 51 & $40-63$ & 53 & $40-64$ \\
\hline Postmenopausal (\%) & & 43 & & - \\
\hline \multicolumn{5}{|l|}{ Behavioural } \\
\hline University degree (\%) & & 24 & & 28 \\
\hline Current smoker (\%) & & 18 & & 29 \\
\hline Physically active (\%) & & 35 & & 47 \\
\hline \multicolumn{5}{|l|}{ Dietary intake } \\
\hline Total energy intake (MJ) & $7 \cdot 9$ & $5 \cdot 5-11 \cdot 2$ & $10 \cdot 0$ & $7 \cdot 0-13.9$ \\
\hline Total fish (g/d) & 29 & $5-81$ & 29 & $5-82$ \\
\hline Lean fish $(\mathrm{g} / \mathrm{d})$ & 13 & $0-49$ & 14 & $0-44$ \\
\hline Fatty fish $(g / d)$ & 8 & $0-30$ & 8 & $0-37$ \\
\hline \multicolumn{5}{|l|}{ Anthropometric } \\
\hline Height at enrolment (m) & 1.62 & $1.54-1.71$ & 1.77 & $1.69-1.85$ \\
\hline Weight at enrolment (kg) & 64 & $53-80$ & 80 & $67-96$ \\
\hline BMI at enrolment $\left(\mathrm{kg} / \mathrm{m}^{2}\right)$ & 24 & $20-30$ & 26 & $22-31$ \\
\hline 1-Year body-weight change (g) & 231 & $-837-1326$ & 119 & $-1058-1176$ \\
\hline Corrected 1-year body-weight change $(\mathrm{g}) \dagger$ & 378 & $-598-1511$ & 444 & $-620-1568$ \\
\hline
\end{tabular}

associated with weight change. Using calibrated fish consumption data did not systematically change the observed associations, but the $95 \%$ CI became wider (Table 2). The results in subgroups from analyses stratified by age at enrolment (Table 3) or BMI at enrolment (Table 4) were not systematically different. Among women, statistically significant heterogeneity across countries was observed for total fish consumption $\left(I^{2}=86.0 \%, \quad P=0.000 ; \quad\right.$ Fig. S2, available online), lean fish consumption $\left(I^{2}=61 \cdot 6 \%, P=0.001\right.$; Fig. S3, available online) and fatty fish consumption $\left(I^{2}=80 \cdot 0 \%\right.$,

Table 2. Association between fish consumption (10 g/d) and 1-year change in body weight $(\mathrm{g})$ ( $\beta$ Coefficients and $95 \%$ confidence intervals)

\begin{tabular}{|c|c|c|c|c|}
\hline & \multicolumn{2}{|c|}{ Women $(\mathrm{g} / 10 \mathrm{~g})$} & \multicolumn{2}{|c|}{ Men $(\mathrm{g} / 10 \mathrm{~g})$} \\
\hline & $\beta$ & $95 \% \mathrm{Cl}$ & $\beta$ & $95 \% \mathrm{Cl}$ \\
\hline \multicolumn{5}{|l|}{ Total fish* } \\
\hline Model $1 \dagger$ & 1.84 & $0.50,3 \cdot 18$ & -5.79 & $-7.94,-3.63$ \\
\hline Model $2 \ddagger$ & $5 \cdot 70$ & $4.35,7.06$ & -1.81 & $-3.96,0.33$ \\
\hline Model 2 (calibrated data)§ & $15 \cdot 19$ & $7 \cdot 80,19.72$ & -3.69 & $-8 \cdot 18,1.74$ \\
\hline \multicolumn{5}{|l|}{ Lean fish\| } \\
\hline Model 1† & $-2 \cdot 76$ & $-4.85,-0.67$ & -7.52 & $-11.04,-4.00$ \\
\hline Model $2 \ddagger$ & $2 \cdot 23$ & $0 \cdot 16,4.31$ & -0.73 & $-4 \cdot 17,2 \cdot 71$ \\
\hline Model 2 (calibrated data)§ & 1.46 & $0.48,16.98$ & 0.04 & $-6 \cdot 80,6 \cdot 19$ \\
\hline \multicolumn{5}{|l|}{ Fatty fish\| } \\
\hline Model $1 \dagger$ & $7 \cdot 78$ & $4 \cdot 82,10 \cdot 75$ & -3.06 & $-6.99,0.86$ \\
\hline Model $2 \ddagger$ & $11 \cdot 12$ & $8.17,14.08$ & -0.25 & $-4 \cdot 10,3.60$ \\
\hline Model 2 (calibrated data)§ & $7 \cdot 57$ & $-1 \cdot 66,15 \cdot 61$ & -1.67 & $-8 \cdot 85,6 \cdot 16$ \\
\hline
\end{tabular}

* $n 249558$ for women and $n 95199$ for men.

†From multilevel mixed-effects linear regression with random effects on intercept, taking into account the clustering of the data within centres nested within countries plus adjusting for an indicator variable for fish consumption. Lean and fatty fish consumptions were analysed in mutually adjusted models.

$\ddagger$ As model 1 plus adjusting for age, BMI at enrolment, menopausal status (women only), highest educational level achieved, smoking status, physical activity level, total energy intake and an indicator variable for the plausibility of reported energy intake.

$\S$ In order to adjust for potential systematic over- or underestimation of fish consumption measurements by the country-specific dietary questionnaires, the analyses were rerun using predicted fish consumption data obtained from the regression of the standardised $24 \mathrm{~h}$ dietary recall interview measurements on the country-specific dietary questionnaire measurements. Covariates as listed in model 2, the season of the year in which the $24 \mathrm{~h}$ dietary recall interview was collected, and an interaction term between centre and dietary questionnaire were included in the model. The standard errors of the coefficients were estimated using bootstrap sampling (300 loops).

$\| n 222609$ for women and $n 77219$ for men. 
Table 3. Association between fish consumption $(10 \mathrm{~g} / \mathrm{d})$ and 1-year change in body weight $(\mathrm{g})$ stratified by age at enrolment ( $\beta$ Coefficients and $95 \%$ confidence intervals)

\begin{tabular}{|c|c|c|c|c|c|c|c|c|}
\hline & \multicolumn{4}{|c|}{ Women $(\mathrm{g} / 10 \mathrm{~g})$} & \multicolumn{4}{|c|}{ Men $(g / 10 \mathrm{~g})$} \\
\hline & \multicolumn{2}{|c|}{$<60$ years } & \multicolumn{2}{|c|}{$\geq 60$ years } & \multicolumn{2}{|c|}{$<60$ years } & \multicolumn{2}{|c|}{$\geq 60$ years } \\
\hline & $\beta$ & $95 \% \mathrm{Cl}$ & $\beta$ & $95 \% \mathrm{Cl}$ & $\beta$ & $95 \% \mathrm{Cl}$ & $\beta$ & $95 \% \mathrm{Cl}$ \\
\hline \multicolumn{9}{|l|}{ Total fish* } \\
\hline Model $1 \dagger$ & $2 \cdot 61$ & $1 \cdot 17,4 \cdot 05$ & 2.02 & $-1.61,5.65$ & $-4 \cdot 41$ & $-6.88,-1.95$ & -8.01 & $-12 \cdot 40,-3.63$ \\
\hline Model 2‡ & $5 \cdot 88$ & $4 \cdot 42,7.35$ & 4.96 & $1 \cdot 31,8.61$ & -0.97 & $-3.44,1.49$ & -4.67 & $-9.01,-0.32$ \\
\hline Model 2 (calibrated data)§ & 18.64 & $7 \cdot 48,21 \cdot 80$ & 7.43 & $-1 \cdot 26,12 \cdot 42$ & -1.74 & $-7.50,2.98$ & -5.90 & $-10.78,3.15$ \\
\hline \multicolumn{9}{|l|}{ Lean fish\| } \\
\hline Model 1† & -0.94 & $-3 \cdot 16,1 \cdot 28$ & $-2 \cdot 37$ & $-8.49,3.74$ & $-6 \cdot 17$ & $-10 \cdot 18,-2 \cdot 17$ & -5.47 & $-12 \cdot 75,1 \cdot 80$ \\
\hline Model $2 \ddagger$ & $2 \cdot 27$ & $0.05,4.49$ & 0.84 & $-5 \cdot 19,6 \cdot 86$ & -1.04 & $-4.97,2.90$ & 0.59 & $-6.49,7.66$ \\
\hline Model 2 (calibrated data)§ & 1.47 & $0.50,17 \cdot 81$ & $5 \cdot 14$ & $-5.85,11.92$ & 0.74 & $-6 \cdot 60,6.68$ & 1.46 & $-9 \cdot 23,12.06$ \\
\hline \multicolumn{9}{|l|}{ Fatty fish\| } \\
\hline Model 1† & 8.46 & $5.23,11.69$ & $6 \cdot 42$ & $-0.92,13.76$ & 0.35 & $-4 \cdot 18,4 \cdot 89$ & -9.24 & $-16.99,-1.49$ \\
\hline Model $2 \ddagger$ & 12.08 & $8 \cdot 85,15 \cdot 32$ & $7 \cdot 37$ & $0.09,14.66$ & $3 \cdot 12$ & $-1.35,7.59$ & -9.90 & $-17.47,-2.32$ \\
\hline Model 2 (calibrated data)§ & 4.99 & $-5.07,14.06$ & 3.66 & $-3.07,17.37$ & -1.87 & $-10.85,9.52$ & $-4 \cdot 15$ & $-13 \cdot 15,12 \cdot 40$ \\
\hline
\end{tabular}

* $n 206508$ for women aged $<60$ years and $n 43050$ for women aged $\geq 60$ years; $n 74727$ for men aged $<60$ years and $n 20472$ for men aged $\geq 60$ years.

†From multilevel mixed-effects linear regression with random effects on intercept, taking into account the clustering of the data within centres nested within countries plus adjusting for an indicator variable for fish consumption. Lean and fatty fish consumptions were analysed in mutually adjusted models.

$\ddagger$ As model 1 plus adjusting for age, BMI at enrolment, menopausal status (women only), highest educational level achieved, smoking status, physical activity level, total energy intake and an indicator variable for the plausibility of reported energy intake.

$\S$ In order to adjust for potential systematic over- or underestimation of fish consumption measurements by the country-specific dietary questionnaires, the analyses were rerun using predicted fish consumption data obtained from the regression of the standardised $24 \mathrm{~h}$ dietary recall interview measurements on the country-specific dietary questionnaire measurements. Covariates as listed in model 2, the season of the year in which the $24 \mathrm{~h}$ dietary recall interview was collected, and an interaction term between centre and dietary questionnaire were included in the model. The standard errors of the coefficients were estimated using bootstrap sampling (300 loops).

$\| n 183236$ for women aged $<60$ years and $n 39373$ for women aged $\geq 60$ years; $n 59945$ for men aged $<60$ years and $n 17274$ for men aged $\geq 60$ years.

$P=0 \cdot 000 ;$ Fig. S4, available online). In most countries, a positive association between fish consumption and weight change was observed. However, in Greece, The Netherlands (Amsterdam/ Maastricht) and the UK (Oxford general population), negative associations for total fish consumption were observed; in Denmark, Greece, The Netherlands (Doetinchem) and the UK (Oxford general population), negative associations for lean fish consumption were observed; and in Sweden (Malmö), a negative association for fatty fish consumption was observed. Among men, heterogeneity across countries was not observed (Figs. S5-S7, available online).

The results from secondary analyses of odds of becoming overweight or obese in 5 years associated with fish consumption $(10 \mathrm{~g} / \mathrm{d})$ are shown in Table 5 . Among women, the OR of becoming overweight was $1.02 / 10 \mathrm{~g}$ higher total fish consumption per d (95\% CI 1.01, 1.02), 1.01/10 g higher lean fish consumption per d (95\% CI $1.00,1.02)$ and $1.02 / 10 \mathrm{~g}$ higher fatty fish consumption per d (95\% CI 1.01, 1.04), after adjustment for potential confounders. Among men, fish consumption was not statistically significantly associated with odds of becoming overweight or obese in 5 years. Among women, statistically significant heterogeneity across countries was observed for total fish consumption ( $I^{2}=66 \cdot 2 \%, P=0 \cdot 000 ;$ Fig. S8, available online), lean fish consumption $\left(I^{2}=54.2 \%, P=0.008\right.$; Fig. S9, available online) and fatty fish consumption $\left(I^{2}=64 \cdot 2 \%, P=0 \cdot 001\right.$; Fig. S10, available online). In most countries, a positive association between fish consumption and becoming overweight or obese was observed. However, in Denmark, Greece, The Netherlands (Utrecht) and the UK (Oxford general population), negative associations for total fish consumption were observed; in Greece, The Netherlands (Amsterdam/Maastricht and Utrecht) and the UK (Oxford general population), negative associations for lean fish consumption were observed; and in Denmark, Norway, Sweden (Malmö) and the UK (Oxford general population), negative associations for fatty fish consumption were observed. Among men, statistically significant heterogeneity across countries was observed for lean fish consumption $\left(I^{2}=46 \cdot 1 \%, P=0 \cdot 046\right.$; Fig. S12, available online) but not for total fish (Fig. S11, available online) or fatty fish consumption (Fig. S13, available online). In most countries, a negative association between lean fish consumption and becoming overweight or obese was observed. However, in The Netherlands (Doetinchem), Sweden (Umeå) and the UK (Oxford health-conscious population), positive associations for lean fish consumption were observed. The results in subgroups from analyses stratified by followup time were not systematically different (data not shown).

\section{Discussion}

In the present study, with participants from ten European countries, fish consumption was weakly positively associated with subsequent change in body weight, but among women only. Expressed in terms of portion sizes, the annual weight change was $11.40 \mathrm{~g}$ per one portion size $(140 \mathrm{~g})$ higher total fish consumption per week (95\% CI 8.69, 14.12). The weak association is thus without any implications at the individual level and probably with minor, if any, implications at the population level.

We used data from different populations with different diets which allowed us to investigate the association between fish 
Table 4. Association between fish consumption (10 g/d) and 1-year change in body weight $(\mathrm{g})$ stratified by BMl at enrolment ( $\beta$ Coefficients and $95 \%$ confidence intervals)

\begin{tabular}{|c|c|c|c|c|c|c|c|c|c|c|c|c|}
\hline & \multicolumn{6}{|c|}{ Women $(\mathrm{g} / 10 \mathrm{~g})$} & \multicolumn{6}{|c|}{ Men $(\mathrm{g} / 10 \mathrm{~g})$} \\
\hline & \multicolumn{2}{|c|}{18.5 to $<25 \mathrm{~kg} / \mathrm{m}^{2}$} & \multicolumn{2}{|c|}{25 to $<30 \mathrm{~kg} / \mathrm{m}^{2}$} & \multicolumn{2}{|c|}{18.5 to $<25 \mathrm{~kg} / \mathrm{m}^{2}$} & \multicolumn{2}{|c|}{$\geq 30 \mathrm{~kg} / \mathrm{m}^{2}$} & \multicolumn{2}{|c|}{25 to $<30 \mathrm{~kg} / \mathrm{m}^{2}$} & \multicolumn{2}{|c|}{$\geq 30 \mathrm{~kg} / \mathrm{m}^{2}$} \\
\hline & $\beta$ & $95 \% \mathrm{Cl}$ & $\beta$ & $95 \% \mathrm{Cl}$ & $\beta$ & $95 \% \mathrm{Cl}$ & $\beta$ & $95 \% \mathrm{Cl}$ & $\beta$ & $95 \% \mathrm{Cl}$ & $\beta$ & $95 \% \mathrm{Cl}$ \\
\hline \multicolumn{13}{|l|}{ Total fish* } \\
\hline Model $1 \dagger$ & 2.67 & $1 \cdot 16,4 \cdot 17$ & 2.86 & $0.15,5.57$ & 0.84 & $-4.34,6.02$ & -3.42 & $-6.86,0.02$ & -4.31 & $-7 \cdot 21,-1.40$ & -4.45 & $-10.50,1.65$ \\
\hline Model $2 \ddagger$ & $5 \cdot 22$ & $3.69,6.76$ & 6.48 & $3.71,9.24$ & 3.70 & $-1.56,9.95$ & -0.42 & $-3.86,3.03$ & $-2 \cdot 15$ & $-5.09,0.78$ & -2.54 & $-8.72,3.64$ \\
\hline Model 2 (calibrated data)§ & $11 \cdot 26$ & $3.28,15 \cdot 56$ & 9.71 & $0.05,14.06$ & 0.54 & $-8.86,14.89$ & 3.94 & $-3.97,8.31$ & -1.75 & $-6.85,2.59$ & -1.29 & $-11.59,3.96$ \\
\hline \multicolumn{13}{|l|}{ Lean fish $\|$} \\
\hline Model $1 \dagger$ & -1.37 & $-3.72,0.98$ & -1.43 & $-5 \cdot 69,2 \cdot 82$ & -1.77 & $-9.97,6.44$ & -7.22 & $-12.95,-1.49$ & -4.50 & $-9.19,0.18$ & -4.88 & $-14.91,5 \cdot 15$ \\
\hline Model $2 \ddagger$ & 1.94 & $-0.42,4.29$ & $3 \cdot 71$ & $-0.55,7.97$ & 0.90 & $-7.24,9.03$ & -1.24 & $-6.89,4.42$ & -0.16 & $-4.82,4.49$ & -0.35 & $-10 \cdot 28,9.57$ \\
\hline Model 2 (calibrated data)§ & 0.93 & $-0.29,12 \cdot 18$ & $6 \cdot 67$ & $-5.45,12.92$ & -0.21 & $-7 \cdot 21,9.40$ & -1.23 & $-3.75,3.11$ & 0.49 & $-4.82,8.01$ & -0.76 & $-8.92,8.80$ \\
\hline \multicolumn{13}{|l|}{ Fatty fish\| } \\
\hline Model $1 \dagger$ & 7.35 & $3.99,10.72$ & 7.91 & $2.00,13.81$ & 9.98 & $-1.93,21.88$ & -1.74 & $-7.59,4.12$ & -0.59 & $-5.92,4.73$ & -6.96 & $-19 \cdot 07,5 \cdot 15$ \\
\hline Model $2 \ddagger$ & 9.58 & $6 \cdot 20,12 \cdot 96$ & 11.45 & $5.53,17.38$ & $12 \cdot 12$ & $0.23,24.01$ & 0.66 & $-5 \cdot 11,6 \cdot 44$ & $1 \cdot 16$ & $-4 \cdot 16,6 \cdot 48$ & -5.50 & $-17.57,6.56$ \\
\hline Model 2 (calibrated data)§ & 5.86 & $-4 \cdot 10,10 \cdot 89$ & $6 \cdot 38$ & $-5.43,15 \cdot 72$ & -5.43 & $-12 \cdot 37,13 \cdot 53$ & -0.47 & $-6 \cdot 37,6 \cdot 89$ & 0.77 & $-8.52,9.75$ & $1 \cdot 21$ & $-4.54,12 \cdot 65$ \\
\hline
\end{tabular}

*There were insufficient data for analysis among persons with BMI $<18.5 \mathrm{~kg} / \mathrm{m}^{2} . n 146177$ for women with BMl of 18.5 to $<25.5 \mathrm{~kg} / \mathrm{m}^{2}, n 70000$ for women with BMI of 25.5 to $<30 \mathrm{~kg} / \mathrm{m}^{2}$, and $n 28750$ for women with BMI of $\geq 30 \mathrm{~kg} / \mathrm{m}^{2} ; n 33353$ for men with BMl of 18.5 to $<25.5 \mathrm{~kg} / \mathrm{m}^{2}, n 47235$ for men with BMl of 25.5 to $<30 \mathrm{~kg} / \mathrm{m}^{2}$, and $n 14301$ for men with BMl of $\geq 30 \mathrm{~kg} / \mathrm{m}^{2}$.

†From multilevel mixed-effects linear regression with random effects on intercept, taking into account the clustering of the data within centres nested within countries plus adjusting for an indicator variable for fish consumption. Lean and fatty fish consumptions were analysed in mutually adjusted models.

₹As model 1 plus adjusting for age, BMI at enrolment, menopausal status (women only), highest educational level achieved, smoking status, physical activity level, total energy intake and an indicator variable for the plausibility of reported energy intake.

$\S$ In order to adjust for potential systematic over- or underestimation of fish consumption measurements by the country-specific dietary questionnaires, the analyses were rerun using predicted fish consumption data obtained from the regression of the standardised $24 \mathrm{~h}$ dietary recall interview measurements on the dietary country-specific questionnaire measurements. Covariates as listed in model 2 , the season of the year in which the $24 \mathrm{~h}$ dietary recall interview was collected, and an interaction term between centre and dietary questionnaire were included in the model. The standard errors of the coefficients were estimated using bootstrap sampling (300 loops).

$\| n 133093$ for women with BMl of 18.5 to $<25.5 \mathrm{~kg} / \mathrm{m}^{2}, n 61043$ for women with BMI of 25.5 to $<30 \mathrm{~kg} / \mathrm{m}^{2}$, and $n 24109$ for women with BMI of $\geq 30 \mathrm{~kg} / \mathrm{m}^{2} ; n 28015$ for men with BMl of 18.5 to $<25.5 \mathrm{~kg} / \mathrm{m}^{2}, n 37641$ for men with BMl of 25.5 to $<30 \mathrm{~kg} / \mathrm{m}^{2}$, and $n 11282$ for men with BMl of $\geq 30 \mathrm{~kg} / \mathrm{m}^{2}$. 
Table 5. Odds of becoming overweight or obese in 5 years associated with fish consumption $(10 \mathrm{~g} / \mathrm{d})$

(Odds ratios and $95 \%$ confidence intervals)

\begin{tabular}{|c|c|c|c|c|}
\hline & \multicolumn{2}{|c|}{ Women } & \multicolumn{2}{|c|}{ Men } \\
\hline & OR & $95 \% \mathrm{Cl}$ & OR & $95 \% \mathrm{Cl}$ \\
\hline \multicolumn{5}{|l|}{ Total fish* } \\
\hline Model $1 \dagger$ & 1.02 & $1.02,1.03$ & 1.00 & $0.99,1.01$ \\
\hline Model $2 \ddagger$ & 1.02 & $1.01,1.02$ & 0.99 & $0.98,1.00$ \\
\hline \multicolumn{5}{|l|}{ Lean fish§ } \\
\hline Model $1 \dagger$ & 1.01 & $1.01,1.02$ & 0.99 & $0.97,1.01$ \\
\hline Model 2ł & 1.01 & $1.00,1.02$ & 0.98 & $0.96,1.00$ \\
\hline \multicolumn{5}{|l|}{ Fatty fish§ } \\
\hline Model $1 \dagger$ & 1.03 & $1.02,1.04$ & 1.00 & $0.98,1,02$ \\
\hline Model 2‡ & 1.02 & $1.01,1.04$ & 1.00 & $0.97,1.02$ \\
\hline
\end{tabular}

${ }^{*} n 150808$ for women and $n 33663$ for men.

†From logistic regression with random effects on intercept, taking into account the clustering of the data within centres nested within countries plus adjusting for an indicator variable for fish consumption. Lean and fatty fish consumptions were analysed in mutually adjusted models.

$\ddagger$ As model 1 plus adjusting for age, BMI at enrolment, menopausal status (women only), highest educational level achieved, smoking status, physical activity level, total energy intake and an indicator variable for the plausibility of reported energy intake.

$\S n 137457$ for women and $n 28296$ for men.

consumption and subsequent change in body weight among women and men and over a wide range of exposures (5-81 g fish/d among women and 5-82 g fish/d among men). We observed statistically significant heterogeneity across studies for several of our analyses. The large study sizes confer a statistical power that allowed us to detect heterogeneity, which is of minor, if any, clinical relevance. Visual inspection of our various forest plots and the relative consistency of the measures of associations across age and BMI at enrolment as well as follow-up time suggest that there is considerable consistency in the findings across studies and across strata. Exclusions were primarily due to nonresponse at follow-up. Non-response may be related to either the exposure to fish consumption or the weight change but most probably not to both the exposure to fish consumption and the weight change. Thus, selection bias is unlikely to have affected the results. Measurement errors may have affected the results. In order to adjust for potential systematic over- or underestimation of fish consumption measurements by the dietary questionnaires, the analyses were rerun using calibrated fish consumption data. This did not, however, systematically change the observed associations, but the $95 \%$ CI became slightly wider. At enrolment, weight was measured by trained staff, but at follow-up, information on weight was self-measured by the participants in most centres. Most probably, however, the reporting of follow-up weight was not related to the exposure to fish consumption. Thus, information bias is of limited concern. Detailed information on the potential confounders reduces the possibility of residual confounding. However, confounding from other risk factors for development of obesity not taken into account remains a possible explanation for the findings. Adjustment for total energy intake has two implications: first, non-energy effects of fish consumption are investigated; second, a substitution model is introduced. Fish consumption and food choices associated with fish consumption may replace food choices, which potentially affect body composition and shape, but in the present study, the replacement foods were not specified. Studies on overall dietary patterns, e.g. the Mediterranean dietary pattern, and weight change are available ${ }^{(20)}$, but these studies do not provide information on the role of individual foods. Thus, investigating dietary patterns and investigating specific foods are complementary approaches towards providing knowledge of how the intake of foods affects body weight. In sensitivity analyses, we included other foods than fish that are characteristic of the Mediterranean dietary pattern. Additional adjustment for intake of these foods did not change the measures of associations. Although, the interpretation of the two models is different, these analyses suggest that fish consumption is not just an indicator of other foods which may be important in body composition and shape.

The principal long-chain $n-3$ PUFA in the diet are EPA and DHA, with fish consumption being the main source of longchain n-3 PUFA. In a clinical trial, the substitution of fish oil capsules for visible fats (butter, olive oil, sunflower oil and peanut oil) statistically significantly decreased body fat mass among healthy young adults ${ }^{(21)}$. Furthermore, substitution of fish oil for visible fats increased basal fat oxidation, suggesting that long-chain $n-3$ PUFA may to some extent modulate the balance between fat oxidation and deposition. In a weightloss trial, however, supplemental long-chain $n-3$ PUFA did not increase loss of body fat mass or weight among overweight and obese adults ${ }^{(22)}$.

The findings from the present study are in agreement with previous findings of no appreciable associations between fish consumption and subsequent change in body weight among middle-aged women and men ${ }^{(3,4)}$ but are in contrast with the findings from a clinical trial of young overweight or obese adults following an energy-restricted diet for 8 weeks $^{(5)}$. In that trial, the inclusion of lean fish, fatty fish or fish oil capsules resulted in statistically significantly more weight loss than did a control diet without marine food, but among men only.

The association between fish consumption as a proxy measure for intake of long-chain n-3 PUFA and body-weight change may depend on the size of the fat mass at enrolment. In the present study, fish consumption was weakly positively associated with subsequent weight change among women but not among men. However, the present study did not suggest systematical differences in the associations across strata of age or across strata of general obesity measured as BMI.

In conclusion, the present study provides strong evidence to support that fish consumption does not prevent body-weight gain. However, the absence of weight change may imply important changes in body composition and shape. Future studies should address these aspects before recommendations on fish consumption can be made. We have investigated the association between fish consumption and subsequent change in waist circumference ${ }^{(23)}$. That study provided evidence to support that fish consumption does not prevent an increase in waist circumference. 


\section{Acknowledgements}

This study is part of the project PANACEA, which is supported by the European Union in the framework of the Public Health Programme (contract 2005328). The study was further supported by the European Commission: Public Health and Consumer Protection Directorate 1993-2004; Research Directorate-General 2005; Ligue contre le Cancer, Societé 3M, Mutuelle Générale de l'Education Nationale, Institut National de la Santé de la Recherche Médicale (INSERM) (France); German Cancer Aid, German Cancer Research Center, Federal Ministry of Education and Research (Germany); Danish Cancer Society (Denmark); Health Research Fund (FIS) of the Spanish Ministry of Health, the participating regional governments and institutions, The ISCIII of the Spanish Ministry of Health (RETICC DR06/0020) (Spain); Cancer Research UK, Medical Research Council, Stroke Association, British Heart Foundation, Department of Health, Food Standards Agency, the Wellcome Trust (UK); Greek Ministry of Health and Social Solidarity, Hellenic Health Foundation and Stavros Niarchos Foundation (Greece); Italian Association for Research on Cancer, National Research Council (Italy); Dutch Ministry of Public Health, Welfare and Sports, Dutch Ministry of Health, Dutch Prevention Funds, LK Research Funds, Dutch ZON (Zorg Onderzoek Nederland), World Cancer Research Fund (WCRF) (The Netherlands); Swedish Cancer Society, Swedish Scientific Council, Regional Government of Skåne and Västerbotten (Sweden); the Norwegian Cancer Society (Norway). Further, this study is part of the project Hepatic and Adipose Tissue and Functions in the Metabolic Syndrome (HEPADIP, www.hepadip.org), which is supported by the European Commission as an Integrated Project under the sixth Framework Programme (contract LSHM-CT-2005-018734) and part of the research programme of the Danish Obesity Research Centre (DanORC, www.danorc.dk), which is supported by the Danish Council for Strategic Research (contract 2101-060005). None of the authors had any conflicts of interest. M. U. J., P. H. M. P. and K. O. designed the study; A. Tj., F. C.-C., H. B., A. Tr., D. P., H. B. B.-d.-M., G. H., K.-T. K., N. J. W., T. J. K., P. H. M. P. and K. O. collected the data; C. D. and K. M. D. performed the statistical analyses; M. U. J., C. D., K. M. D., A. M. M., D. R., A.-C. V., T. N., T. I. A. S., J. H., A. Tj., M.-C. B.-R., F. C.-C., G. F., B. T., T. K., M. M. B., H. B., A. N., P. O., A. Tr., D. P., M. S. D. M., S. S., H. B. B.-d.-M., D. L. v. d. A., D. E., A. H., L. R., A. A., E. M.-M., J. M. H., A. B., P. A., J. M., E. W., G. H., I. J., K.-T. K., N. J. W., T. J. K., V. C., N. S., E. R., P. H. M. P. and K. O. wrote the paper; M. U. J. had primary responsibility for the final content. All authors read and approved the final manuscript.

Supplementary figures and table are available online at http://www.journals.cambridge.org/bjn

\section{References}

1. Buckley JD \& Howe PR (2009) Anti-obesity effects of longchain omega-3 polyunsaturated fatty acids. Obes Rev 10, 648-659.

2. Liaset B, Madsen L, Hao Q, et al. (2009) Fish protein hydrolysate elevates plasma bile acids and reduces visceral adipose tissue mass in rats. Biochim Biophys Acta 1791, 254-262.

3. Schulz M, Kroke A, Liese AD, et al. (2002) Food groups as predictors for short-term weight changes in men and women of the EPIC-Potsdam cohort. J Nutr 132, 1335-1340.

4. Sanchez-Villegas A, Bes-Rastrollo M, Martinez-Gonzalez MA, et al. (2006) Adherence to a Mediterranean dietary pattern and weight gain in a follow-up study: the SUN cohort. Int J Obes 30, 350-358.

5. Thorsdottir I, Tomasson H, Gunnarsdottir I, et al. (2007) Randomized trial of weight-loss-diets for young adults varying in fish and fish oil content. Int J Obes 31, 1560-1566.

6. Riboli E \& Kaaks R (1997) The EPIC Project: rationale and study design. European Prospective Investigation into Cancer and Nutrition. Int J Epidemiol 26, Suppl. 1, S6-S14.

7. Riboli E, Hunt KJ, Slimani N, et al. (2002) European Prospective Investigation into Cancer and Nutrition (EPIC): study populations and data collection. Public Health Nutr 5, 1113-1124.

8. Kaaks R, Slimani N \& Riboli E (1997) Pilot phase studies on the accuracy of dietary intake measurements in the EPIC project: overall evaluation of results. European Prospective Investigation into Cancer and Nutrition. Int J Epidemiol 26, Suppl. 1, S26-S36.

9. Welch AA, Lund E, Amiano P, et al. (2002) Variability of fish consumption within the 10 European countries participating in the European Investigation into Cancer and Nutrition (EPIC) study. Public Health Nutr 5, 1273-1285.

10. Slimani N, Kaaks R, Ferrari P, et al. (2002) European Prospective Investigation into Cancer and Nutrition (EPIC) calibration study: rationale, design and population characteristics. Public Health Nutr 5, 1125-1145.

11. Slimani N, Deharveng G, Unwin I, et al. (2007) The EPIC nutrient database project (ENDB): a first attempt to standardize nutrient databases across the 10 European countries participating in the EPIC study. Eur J Clin Nutr 61, $1037-1056$.

12. Haftenberger M, Lahmann PH, Panico S, et al. (2002) Overweight, obesity and fat distribution in 50- to 64-year-old participants in the European Prospective Investigation into Cancer and Nutrition (EPIC). Public Health Nutr 5, 1147-1162.

13. de Groot LC, Sette S, Zajkas G, et al. (1991) Nutritional status: anthropometry. Euronut SENECA investigators. Eur J Clin Nutr 45, Suppl. 3, S31-S42.

14. Spencer EA, Appleby PN, Davey GK, et al. (2002) Validity of self-reported height and weight in 4808 EPIC-Oxford participants. Public Health Nutr 5, 561-565.

15. Keys A, Fidanza F, Karvonen MJ, et al. (1972) Indices of relative weight and obesity. J Chronic Dis 25, 329-343.

16. Wareham NJ, Jakes RW, Rennie KL, et al. (2003) Validity and repeatability of a simple index derived from the short physical activity questionnaire used in the European Prospective Investigation into Cancer and Nutrition (EPIC) study. Public Health Nutr 6, 407-413.

17. Goldberg GR, Black AE, Jebb SA, et al. (1991) Critical evaluation of energy intake data using fundamental principles of energy physiology: 1 . Derivation of cut-off limits to identify under-recording. Eur J Clin Nutr 45, 569-581.

18. Black AE (2000) Critical evaluation of energy intake using the Goldberg cut-off for energy intake:basal metabolic rate. A practical guide to its calculation, use and limitations. Int J Obes 24, 1119-1130.

19. Ferrari P, Day NE, Boshuizen HC, et al. (2008) The evaluation of the diet/disease relation in the EPIC study: considerations for the calibration and the disease models. Int J Epidemiol 37, 368-378. 
20. Romaguera D, Norat T, Vergnaud AC, et al. (2010) Mediterranean dietary patterns and prospective weight change in participants of the EPIC-PANACEA project. Am J Clin Nutr 92, 912-921.

21. Couet C, Delarue J, Ritz P, et al. (1997) Effect of dietary fish oil on body fat mass and basal fat oxidation in healthy adults. Int J Obes 21, 637-643.
22. DeFina LF, Marcoux LG, Devers SM, et al. (2011) Effects of omega-3 supplementation in combination with diet and exercise on weight loss and body composition. Am J Clin Nutr 93, 455-462.

23. Jakobsen MU, Due KM, Dethlefsen C, et al. (2012) Fish consumption does not prevent increase in waist circumference in European women and men. Br J Nutr 108, 924-931. 